Patients had been randomly allocated to treatment with either nilutamide $(300 \mathrm{mg} /$ day for the first month then $150 \mathrm{mg} /$ day thereafter) or a vaccination regimen that comprised a priming immunization with two admixed recombinant vaccinia-based vaccines on the first day (one with transgenes for PSA and the other with trangenes for the human T-cell costimulatory molecule B7-1) followed by monthly boosts with a recombinant fowlpoxbased PSA vaccine. Patients were monitored monthly, and those who showed an increase in serum PSA but no evidence of metastatic disease were crossed over to receive both nilutamide and vaccination.

At a median potential follow-up of 4.4 years, overall survival from time of enrollment was greater in patients who received the vaccination regimen $(n=21)$ than in those treated with nilutamide ( $n=21$; median 5.1 years vs 3.4 years). Of the 20 patients who crossed over to combined therapy, those who were initially in the vaccination arm $(n=12)$ had significantly longer overall survival than patients who started out in the nilutamide arm $(n=8$; median 6.2 years vs 3.7 years from the on-study date; $P=0.045$ ).

The authors suggest that patients with nonmetastatic, castration-resistant prostate cancer would benefit from vaccination early in their treatment program.

Original article Madan RA et al. (2008) Analysis of overall survival in patients with nonmetastatic castration-resistant prostate cancer treated with vaccine, nilutamide, and combination therapy. Clin Cancer Res 14: 4526-4531

\section{Serum calcium levels might predict the development of prostate cancer}

Men with elevated serum calcium levels might have an increased risk of fatal prostate cancer, according to a new American study. Previous research has shown that both calcium and parathyroid hormone, which controls serum calcium levels, can promote growth and metastasis of prostate cancer cells.

Skinner and Schwartz analyzed data from 2,814 men (aged 24-77 years at baseline) enrolled in the National Health and Nutrition Examination Survey (NHANES). Serum calcium measurements were taken at baseline. Over a follow-up period of 46,188 person years, 85 incident cases of prostate cancer and 25 prostate cancer deaths occurred. On dividing these cases into three groups according to serum calcium levels, the researchers found that men in the upper tertile (mean serum calcium $10.2 \pm 0.3 \mathrm{mg} / \mathrm{dl}$ ) had nearly three times the risk of fatal prostate cancer compared with men in the middle and lower tertiles (mean serum calcium levels $9.7 \pm 0.1 \mathrm{mg} / \mathrm{dl}$ and $9.3 \pm 0.3 \mathrm{mg} / \mathrm{dl}$, respectively). No such association was identified for incident prostate cancer.

According to the authors, these results suggest that serum calcium levels are comparable to family history in predicting the risk for fatal prostate cancer. They explain that these findings could be important because, unlike family history, serum calcium level is a risk factor that can be controlled with medication. Skinner and Schwartz added that, owing to the small number of fatal cases in the present study, further prospective studies are required to confirm these findings.

Original article Skinner HG and Schwartz GG (2008)

Serum calcium and incident and fatal prostate cancer in the National Health and Nutrition Examination Survey. Cancer Epidemiol Biomarkers Prev 17: 2302-2305

\section{Patients with bladder cancer have good short-term outcomes after robotic radical cystectomy}

Case studies have reported good surgical and perioperative outcomes for robotic radical cystectomy in patients with bladder cancer; however, there is little information on shortterm postoperative outcomes. To address this issue, Pruthi and Wallen analyzed the outcomes of 50 patients with localized urothelial carcinoma of the bladder who had undergone robot-assisted laparoscopic radical cystectomy and extracorporeal urinary diversion.

No instances of a positive surgical margin were recorded at the time of operative pathological examination. Cystectomy specimens taken during surgery showed that $33(66 \%)$ patients had organ-confined disease (pathologic stage pT2 or less), 7 (14\%) had localized extravesical disease (stage T3), and 10 (20\%) had node-positive disease.

After a mean clinical follow-up period of 13.2 months (range 2-24 months), 7 (14\%) patients -all of whom had pT2 or pT3 disease -had evidence of recurrent disease. Three 